\title{
ÉTUDE COMPARÉE DE LA DIGESTIBILITÉ DU SON DE FROMENT PAR DIVERSES MÉTHODES
}

PAR

\author{
G. CHARLET-LERY et A. M. LEROY
}

Laboratoire de Recherches Zootechniques, Institut National Agronomique. Paris

La méthode classique de détermination des coefficients de digestibilité d'un aliment qui ne peut pas être consommé seul, ce qui oblige à le faire ingérer en même temps qu'une quantité connue d'un aliment de référence, suppose implicitement que la digestibilité de ce dernier reste constante dans tous les cas, quelles que soient les proportions relatives et le choix des deux aliments associés.

A cette hypothèse s'opposent les constatations de nombreux chercheurs concernant les phénomènes de digestibilité associative. Cependant, il est possible que, tant que certains équilibres nutritionnels ne sont pas rompus, les variations dues à ces phénomènes soient difficilement mises en évidence, par suite des erreurs expérimentales elles-mêmes. C'est, i1 nous semble, ce que nous avons observé en étudiant la digestibilité de la pulpe sèche de betterave, chez les bovins et les ovins. Au cours de ces expériences (I), la moyenne des coefficients de digestibilité de la matière organique de la pulpe sèche de betterave, après réhumidification, s'est élevée à $76, \mathrm{I} \pm 4,4$ lorsque nous l'avons calculée à partir de 12 périodes de régime mixte où l'aliment de référence était le foin de luzerne et à $76,9 \pm 0,6$ lorsque nous l'avons observée directement sur 3 brebis soumises à un régime exclusif de pulpe.

Il semble difficile, actuellement, d'éviter l'emploi de la méthode précitée, mais les modalités d'application pratique des calculs, suivant la façon dont on les conduit, n'aboutissent pas aux mêmes résultats.

I. - Dans le calcul classique, connaissant préalablement les coefficients de digestibilité des divers principes de l'aliment de référence, nous les appliquons dans le régime mixte aux principes correspondants, ce qui nous permet, par différence, de calculer la quantité d'éléments digérés relatifs à 1'aliment étudié.

Les résultats dépendent donc partiellement des valeurs trouvées pour les coefficients de digestibilité de l'aliment de référence; mais, les erreurs prennent une importance d'autant plus grande que le rapport 
$\frac{\text { principe dans l'aliment étudié }}{\text { principe dans le régime mixte }}$ est faible, comme nous avons pu le constater au cours des études sur la pulpe de betterave desséchée oì, par suite de l'apport très faible d'azote par la pulpe, les résultats sont très hétérogènes, ce qui entraîne une erreur type sur le coefficient de digestibilité de l'azote de ro,o pour une valeur de 36,2 .

Ce procédé de calcul oblige à multiplier les expériences pour obtenir des coefficients de digestibilité moyens ayant une valeur certaine et il exige que l'on incorpore des quantités aussi importantes que possible de l'aliment étudié dans le régime mixte, ce qui n'est pas toujours possible. La dispersion parfois très grande des résultats rend difficile leur interprétation.

II. - La méthode de CORBERy et ChatTERJEE, exposé par SCHURCh (2) reprise par GASNIER et VACHEL (3) n'oblige pas à se référer à un régime de référence; de ce fait, on réduit les limites entre lesquelles on émet 1'hypothèse que les coefficients de digestibilité des constituants d'une ration mixte restent constants.

Pour chaque régime mixte, il est possible d'écrire que la digestibilité de la ration totale est la somme pondérée des digestibilités des 2 composants en cause. Si $x$ et $y$ représentent ces coefficients de digestibilité, A et $\mathrm{B}$ les pourcentages des composants, 1'équation s'écrit :

$$
\mathrm{Ax}+\mathrm{By}=\mathrm{D}
$$

$D$, étant le coefficient de digestibilité de la ration totale.

A condition de considérer les expériences par groupe de 2 , il est facile de résoudre ce système d'équations pour obtenir les valeurs de $x$ et, éventuellement, de $y$. Ce mode de calcul a l'avantage de ne pas préjuger de la digestibilité du régime de référence et son utilisation devrait permettre de supprimer l'étude de ce dernier régime.

Mais cette méthode revient à extrapoler la droite définie par les couples de points $A$. D (variation du coefficient de digestibilité du régime mixte en fonction du pourcentage de l'aliment étudié introduit dans la ration) pour la valeur $\mathrm{A}=$ Ioo si l'on désire obtenir le coefficient de digestibilité de l'aliment étudié ou pour la valeur $\mathrm{A}=\mathrm{O}$ si on veut calculer celui relatif à l'aliment de référence.

Le calcul des paramètres de la droite et l'extrapolation de celle-ci aux valeurs Ioo et o sont d'exécution rapide.

Si l'aliment étudié a été introduit dans plus de 2 rations mixtes, il est possible d'envisager l'obtention des résultats par 2 procédés.

Io Le premier consiste dans le calcul des coefficients de digestibilité obtenus en groupant les expériences 2 par 2 selon toutes les possibilités et de calculer la moyenne de ces résultats. Puisque, par hypothèse, le phénomène étudié est linéaire, il est possible de grouper les pourcentages extrêmes. 


\section{ERRATA}

\section{ANNALES DE ZOOTECHNIE 1954 \\ (quatrième fascicule)}

Page 346, dans le titre du Tableau II, le terme " grande Calorie " doit être remplacé par "calorie ",

Page 353, le titre "expériences extensives sur le porc " doit être reporté audessus des titres de colonnes qui le précèdent,

même page, le chiffre I4 sera mis à la place du nº I qui précède le titre "Normes danoises »

le chiffre I5 à la place du chiffre 2 qui précède "Normes françaises "

le chiffre 16 à la place du chiffre 3 qui précède "Fxpériences avec avoine ",

Page 354, au lieu de essai No I22, lire essai No I 2 ,

Dans le titre de l'expérience No 2 I lire "calories" au lieu de " grandes calories ",

Page 365, au lieu. de "Borsoock" lire "Borsook",

P'age $366,3^{\mathrm{e}}$ ligne après le tableau, lire "calories" au lieu de "grandes calories $)$,

l'age 367 , le texte à partir de l'équation (I) $\mathrm{R}+m=\mathrm{E}+\mathrm{Ms} \times 09+m s$ $\times \mathrm{O} 9+\mathrm{P}$ jusqu'à la formule $\mathrm{X}=\frac{m^{\prime}-m^{\prime} s}{m-m s}$ doit être remplacé par le suivant :

$\mathrm{I}^{\mathrm{O}}-\mathrm{R}+m=\mathrm{E}+\mathrm{Ms} \times \mathrm{I}, \mathrm{O}+m \mathrm{~s} \times \mathrm{I}, \mathrm{O}+\mathrm{P}$

dans laquelle

$\mathrm{R}$ représente la quantité d'énergie métabolisable de la ration de base, $m$ représente la quantité d'énergie métabolisable apportée par I $\mathrm{kg}$ de 1'Aliment A,

Nis représente la quantité de matière sèche de la ration de base, $m$ s représente la quantité de matière sèche apportée par I kg de l'Aliment A. E représente le besoin d'entretien de l'animal, $P$ représente le besoin de production de ce même animal.

Nous voulons calculer la quantité $X$ d'un aliment $B$, qui, substituée à un $\mathrm{kg}$ de $\mathrm{A}$, continue à maintenir l'animal dans les mêmes conditions, c'est-à-dire qui permet de satisfaire les mêmes besoins d'énergie nette, $\mathrm{E}+\mathrm{P}$.

Appelons $m^{\prime}$ l'apport d'énergie métabolisable par $\mathrm{kg}$ de l'aliment B et $m s^{\prime}$ la quantité correspondante de matière sèche par $\mathrm{kg}$ de $\mathrm{B}$.

La nouvelle équation d'équilibre devient après la substitution :

$2^{0}-\mathrm{R}+m^{\prime} \mathrm{X}=\mathrm{E}+\mathrm{M} s \times \mathbf{I}, \mathrm{O}+m s^{\prime} \mathrm{X} \times \mathrm{I}, \mathrm{O}+\mathrm{P}$

Retranchons membre à membre l'équation (2) de la première, il vient :

$3^{\mathrm{o}}-m-m^{\prime} \mathrm{X} \times \mathrm{I}, \mathrm{O}=m s-m s^{\prime} \mathrm{X} \times \mathrm{I}, \mathrm{O}$

de laquelle nous tirons :

$\mathrm{X}=\frac{m-m s}{m^{\prime}-m s^{\prime}}$

Page 368, Tableau II, colonne énergie métabolisable, retirer les virgules dans les chiffres 2756 calories et 2015 calories.

Même page, Tableau IX : Équivalent de substitution du foin, au lieu de I $550 \mathrm{~kg}$ lire I $603 \mathrm{~kg}$. 


$$
\text { . }
$$


$2^{\circ}$ Le deuxième consiste à calculer les paramètres de la droite de régression définie par l'ensemble des points $\mathrm{A}, \mathrm{D}$.

Si l'on étudie non pas la digestibilité de la matière sèche totale, mais celle d'un des principes de la ration, tel que l'azote par exemple, il est évident que le pourcentage à considérer est le pourcentage de l'azote de l'aliment étudié par rapport à l'azote total du régime.

Dans le travail ci-après, nous avons essayé de comparer entre elles ces diverses méthodes de calcul :

$\mathrm{I}^{0}$ méthode classique de calcul par différence,

$2^{\circ}$ méthode de la droite de régression calculée uniquement à partir des points obtenus sur les régimes mixtes s'il y a plus de 2 données ou droite définie par 2 points s'il n'y a que 2 données.

Grâce à la droite obtenue par cette dernière méthode, nous avons calculé aussi bien les coefficients de digestibilité de l'aliment étudié, le son de froment, que ceux de l'aliment de référence : le foin. Les coefficients de digestibilité du foin, obtenus par ce dernier procédé, ont été comparés aux valeurs obtenues dans les expériences directes.

\section{Protocole expérimental :}

Le protocole expérimental utilisé dans la conduite des expériences de digestibilité a été décrit dans une publication précédente (I).

De mars à juillet I949, nous avons travaillé avec 2 brebis Ile-deFrance, inscrites au Flock-Book de leur race, auxquelles nous avons distribué successivement un régime composé de foin de luzerne, des régimes mixtes où 20,28 et $35 \mathrm{p}$. Ioo de foin ont été remplacés par du son, un second régime de foin de luzerne. De novembre I949 à mars I950, nous avons travaillé avec 2 bœufs Maine-Anjou, qui ont reçu successivement un régime témoin composé de foin de luzerne et des régimes mixtes contenant 26 et 52 p. Ioo de son.

Tous les animaux ont été nourris à volonté et ont maintenu leur poids vif sans gain ou perte appréciable; nous avons essayé de régler au plus près les distributions d'aliments d'après l'appétit des animaux pour éviter le plus possible les refus.

Les périodes d'adaptation aux régimes ont eu des durées minimum de Io jours; les périodes expérimentales ont été poursuivies pendant exactement io jours.

L,es déterminations analytiques effectuées sur les échantillons moyens de foin de luzerne, de refus de foin, de son et de matières fécales ont porté sur la matière sèche, les matières minérales totales, l'azote, les matières organiques, grasses et cellulosiques, ainsi que sur les extractifs non azotés. De plus, nous avons dosé la cellulose pure, la lignine, les pentosanes et les glucides hydrolysables. 
Pour ces dosages, nous avons employé les techniques analytiques généralement utilisées en France (4). La cellulose pure a été dosée par la méthode de Kurschner et HofFER (5) ; la lignine par celle de MAHOOD et CABLE (6) ; les pentosanes par la méthode d'UNGER et JAGER (7) ; les glucides hydrolysables par celle de BERTRAND (8).

\section{Résultats et discussion :}

Le Tableau I donne les résultats d'analyses du foin et du son de froment correspondants aux diverses expériences sur bovins et ovins. Bien que chaque série d'expériences ait été effectuée avec le même stock de foin, nous avons constaté parfois des différences importantes entre les échantillons prélevés au cours de chaque période, surtout dans le cas des ovins, ce qui nous a conduit à prendre comme données de référence pour le calcul par différence, tantôt les résultats de la première, tantôt ceux de la seconde des 2 périodes témoins.

\section{TABLEAU I}

\section{Composition des ingesta}

au cours des diverses périodes expérimentales

en p. 1000 de la matière sèche.

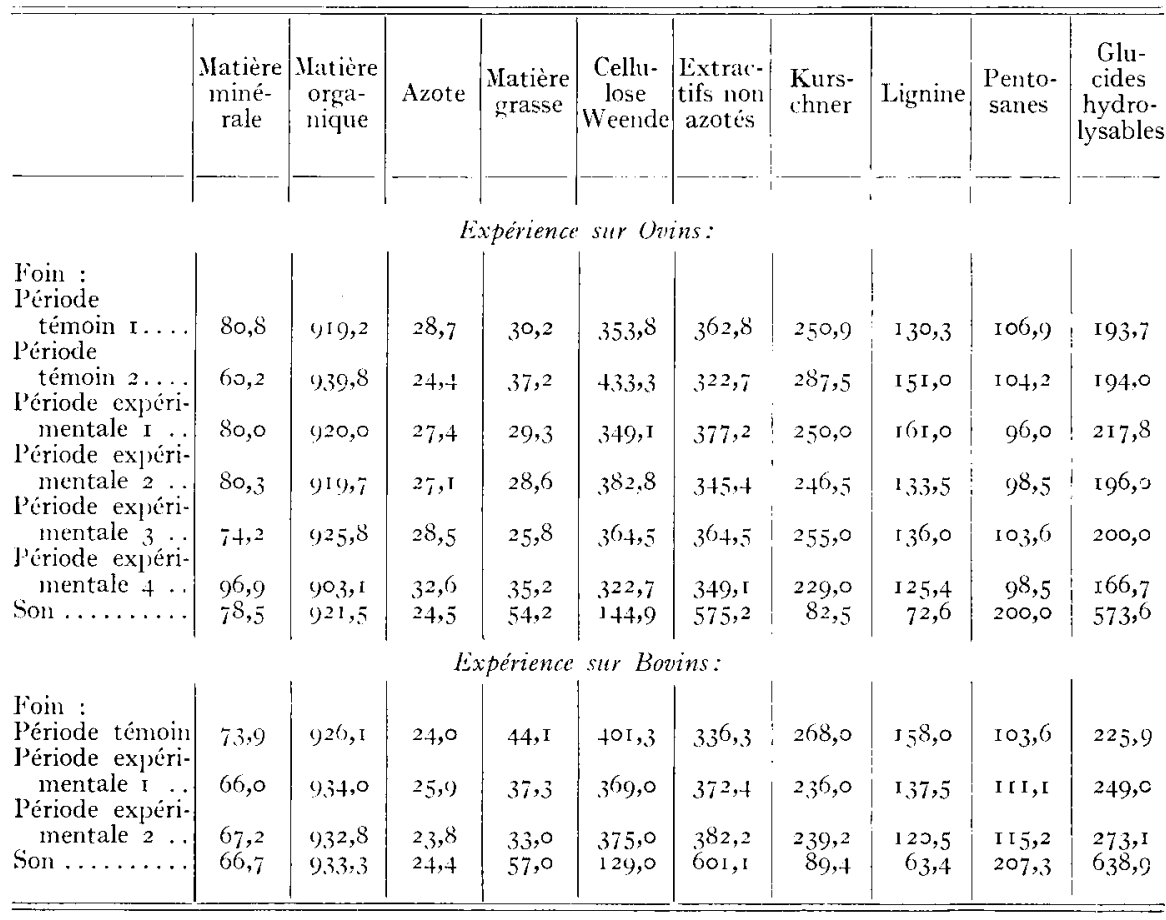


Nous avons tenu compte dans nos calculs des refus de foin dont 1a composition chimique est toujours légèrement différente du foin dont ils proviennent.

En raison de 1'homogénéité des divers échantillons de son, nous avons utilisé, pour nos calculs, les résultats moyens.

Le Tableau II indique, exprimées en matière sèche, les quantités de foin distribuées et refusées, de son ingéré, d'excréta récoltés au cours des périodes expérimentales ainsi que les coefficients de digestibilité correspondants et le pourcentage de la matière sèche du son par rapport à la matière sèche totale ingérée dans le régime de chaque animal.

\section{TABLEAU II}

Ingestion et Excrétion de Matière sèche Coefficient d'utilisation digestive.

\begin{tabular}{|c|c|c|c|c|c|c|c|}
\hline & $\begin{array}{c}\text { Foin } \\
\text { distribué }\end{array}$ & $\begin{array}{l}\text { Foin } \\
\text { refusé }\end{array}$ & $\begin{array}{l}\text { Son } \\
\text { ingéré }\end{array}$ & $\frac{\text { m. s. son }}{\text { m.s. totale }} \times$ roo & $\begin{array}{l}\text { Ingéré } \\
\text { total }\end{array}$ & Excreta & $\mathrm{C}$ u d. \\
\hline \multicolumn{8}{|c|}{ Bovins : } \\
\hline Noir ..... & 10.200 & 967 & $\cdots$ & & 9.233 & $380 \mathrm{I}$ & 58,8 \\
\hline Roux ..... & 10.200 & $\mathrm{I} .5 \mathrm{I} \mathrm{I}$ & 一 & & 8.689 & 3917 & 54,9 \\
\hline \multicolumn{8}{|c|}{ Période expérimentale 1 : } \\
\hline Noir ..... & $6.342,8$ & $\mathrm{I} \cdot 548,7$ & 5280 & $49, \mathrm{I}$ & $10.074, \mathrm{I}$ & 3803 & 62,2 \\
\hline Roux ..... & $6.342,8$ & 1.620 & 5280 & $5^{2,5}$ & $10.002,8$ & 3595 & 64,1 \\
\hline \multicolumn{8}{|c|}{ Période expérimentale II : } \\
\hline Noir...... & 9.240 & $1.917,4$ & 2640 & 26,45 & $9.9^{62.6}$ & 4086,5 & 59,0 \\
\hline Roux...... & 9.240 & $2.084, I$ & 2640 & 26,9 & $9 \cdot 795,9$ & 3899,5 & 60,2 \\
\hline \multicolumn{8}{|c|}{ Ovins: } \\
\hline \multicolumn{8}{|c|}{ Période témoin I : } \\
\hline B $36 \ldots \ldots$ & 1.009 & I34 & - & - & 875 & 370,0 & 57,7 \\
\hline B $37 \ldots \ldots$ & $\mathrm{x} .055$ & I 28 & - & 一 & $9^{2 \%}$ & $3^{89,0}$ & 58,0 \\
\hline \multicolumn{8}{|c|}{ Période témoin II (8 jours seulement) : } \\
\hline B $36 \ldots \ldots$ & $1.057,9$ & 121 & - & - & $93^{6,9}$ & $45^{8,3}$ & $5 \mathrm{I}, \mathrm{I}$ \\
\hline В $37 \ldots \ldots$ & I. I 45 & $62, \mathrm{I}$ & 一 & 一 & I. $.082,9$ & 476,9 & 56,0 \\
\hline \multicolumn{8}{|c|}{ Période expérimentale $I$} \\
\hline B $36 \ldots \ldots 1$ & 890 & 9 & $1 \quad 226$ & $1 \quad 20,4$ & 1.107 & 457 & $5^{8,8}$ \\
\hline \multicolumn{8}{|c|}{ Période expérimentale II } \\
\hline B $36 \ldots \ldots 1$ & $79^{2}$ & $2 \mathrm{I}$ & $3^{\prime} \quad 3^{16,4}$ & $1 \quad 29$ & $\mathrm{r} .087,4$ & 414 & i 62,0 \\
\hline \multicolumn{8}{|c|}{ Période expérimentale III } \\
\hline B $36 \ldots \ldots$ & 744 & 15,7 & 406,8 & 35,6 & I.I $35, \mathrm{I}$ & $45^{6,4}$ & 59,8 \\
\hline B $37 \ldots \ldots$ & 930 & 9,0 & 226 & 20 & I.I 47 & 425,8 & 62,9 \\
\hline \multicolumn{8}{|c|}{ Période expérimentale $I V$ : } \\
\hline B $36 \ldots \ldots$ & 728 & I I, 6 & 406,8 & 35,6 & I. I 23,2 & 389,2 & 65,3 \\
\hline B $37 \ldots \ldots$ & 819 & 6,9 & 316,4 & 28 & I. I 28,5 & 395,0 & 65,0 \\
\hline
\end{tabular}

Le Tableau III fait connaître les coefficients de digestibilité des divers éléments du foin et du son, calculés par différence, ainsi que la 
moyenne de ces résultats et l'erreur type (I). Les coefficients de digestibilité observés chez les bovins sont dans tous les cas, légèrement inférieurs à ceux constatés pour les ovins ; cependant, les différences entre ces 2 espèces n'étant pas signi ficativement différentes, nous avons exprimé le résultat moyen relatif à l'ensemble des sujets.

TABLEAU III

Coefficients de digestibilité des divers éléments du foin et du son (calculés par différence).

\begin{tabular}{|c|c|c|c|c|c|c|c|c|c|c|c|c|}
\hline Animal & $\mid \begin{array}{lr}\text { M. } & \text { s. } \\
\text { son } & \% \\
\text { m. } & \text { s. } \\
\text { totale }\end{array}$ & $\begin{array}{c}\text { Ma- } \\
\text { tière } \\
\text { sèche }\end{array}$ & $\begin{array}{l}\text { Matière } \\
\text { miné- } \\
\text { rale }\end{array}$ & $\begin{array}{l}\text { Ma- } \\
\text { ticre } \\
\text { orga- } \\
\text { nique }\end{array}$ & Azote & $\begin{array}{l}\text { Ma- } \\
\text { tière } \\
\text { gras- } \\
\text { se }\end{array}$ & 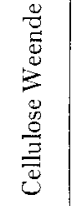 & $\begin{array}{c}\text { Ex- } \\
\text { trac- } \\
\text { tifs } \\
\text { non } \\
\text { azo- } \\
\text { tés }\end{array}$ & $\mid \begin{array}{c}\text { Cel- } \\
\text { lulose } \\
\text { Kurs- } \\
\text { chner }\end{array}$ & Lignine & $\begin{array}{l}\text { Pen- } \\
\text { tosa- } \\
\text { nes }\end{array}$ & $\begin{array}{c}\text { Gluci- } \\
\text { des } \\
\text { hy- } \\
\text { dro- } \\
\text { lysa- } \\
\text { bles }\end{array}$ \\
\hline \multicolumn{13}{|c|}{ Expérience sur Bovins: } \\
\hline 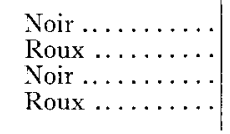 & $\begin{array}{l}26,5 \\
26,7 \\
52,4 \\
52,8\end{array}$ & $\begin{array}{l}65,4 \\
72,2 \\
59,5 \\
74,5\end{array}$ & $\begin{array}{l}38,2 \\
44,6 \\
\text { I } 3,6 \\
55,9\end{array}$ & $\begin{array}{l}67, \mathbf{1} \\
73,8 \\
62, \mathbf{I} \\
75, \mathbf{I}\end{array}$ & $\begin{array}{l}78,6 \\
78,6 \\
68,8 \\
77,8\end{array}$ & $\begin{array}{l}48,0 \\
49,4 \\
19,0 \\
32,5\end{array}$ & $\begin{array}{r}21,7 \\
56,5 \\
-12,0 \\
3,3,8\end{array}$ & $\begin{array}{l}74,2 \\
77,0 \\
74,7 \\
82,8\end{array}$ & $\begin{array}{l}35,7 \\
59,8 \\
14,9 \\
36,3\end{array}$ & $\begin{array}{r}2,5 \\
38,4 \\
-100,5 \\
-69,0\end{array}$ & $\begin{array}{l}71,1 \\
83,4 \\
76,7 \\
93,6\end{array}$ & $\begin{array}{l}90,6 \\
85, \mathrm{I} \\
89, \mathrm{I} \\
95,5\end{array}$ \\
\hline \multicolumn{13}{|c|}{ Expérience sur Ovins: } \\
\hline 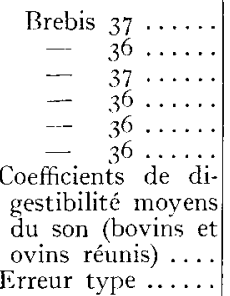 & $\begin{array}{l}20,0 \\
20,4 \\
28,0 \\
29,0 \\
35,6 \\
35,6\end{array}$ & $\begin{array}{l}82,8 \\
62,7 \\
83,0 \\
80,3 \\
63,5 \\
78,8\end{array}$ & $\begin{array}{r}59,2 \\
72,9 \\
59,0 \\
77,5 \\
29,4 \\
77,5\end{array}$ & $\begin{array}{l}89,0 \\
6 \mathrm{I}, 4 \\
85,4 \\
8 \mathrm{I}, 2 \\
65,9 \\
79,5\end{array}$ & $\begin{array}{l}74,5 \\
65,5 \\
91,0 \\
81,8 \\
66,0 \\
86,0\end{array}$ & $\begin{array}{l}55,7 \\
82,7 \\
68,0 \\
69,2 \\
30,0 \\
56,4\end{array}$ & $\begin{array}{r}160,9 \\
65,2 \\
108,8 \\
34,5 \\
67,8 \\
96,5 !\end{array}$ & $\begin{array}{l}75,8 \\
59,2 \\
78,5 \\
94,8 \\
70,3 \\
75,4\end{array}$ & $\begin{array}{r}\text { I } 5 \text { I, I } \\
\text { I } 8,8 \\
\text { I I I, } 5 \\
25,3 \\
64,0 \\
96,0\end{array}$ & $\begin{array}{r}\text { I } 34,7 \\
\text { I } 87,8 \\
77,0 \\
20,4 \\
34,1 \\
80,0\end{array}$ & $\begin{array}{l}78,7 \\
62,4 \\
86,0 \\
96,5 \\
65,5 \\
76,8\end{array}$ & $\begin{array}{l}89,4 \\
77,6 \\
81,6 \\
79,9 \\
80,4 \\
84,4\end{array}$ \\
\hline
\end{tabular}

Le Tableau IV nous permet de comparer les divers modes de calcul et les coefficients auxquels ils permettent d'aboutir : coefficients de digestibilité du son obtenus par différence et par l'intermédiaire de la droite de régression, coefficients de digestibilité du foin, obtenus par différence ou par expérience directe.

Les coefficients de digestibilité obtenus pour le son de troment par ces deux procédés de calcul, ne sont pas, pour chaque élément chimique considéré individuellement, significativement différents. Nous en concluons qu'il est possible d'utiliser dans la pratique les données obtenues par l'une ou l'autre de ces méthodes.

Les moyennes des coefficients de digestibilité calculés grâce aux droites de régression présentent des erreurs types dans l'ensemble plus

(1) Erreur type $: \sqrt{\frac{(x-X)^{2}}{N(N-I)}}$. 


\section{TABLEAU IV}

Coefficients d'utilisation digestive des différents éléments dn son et du foin selon les méthodes de calcul.

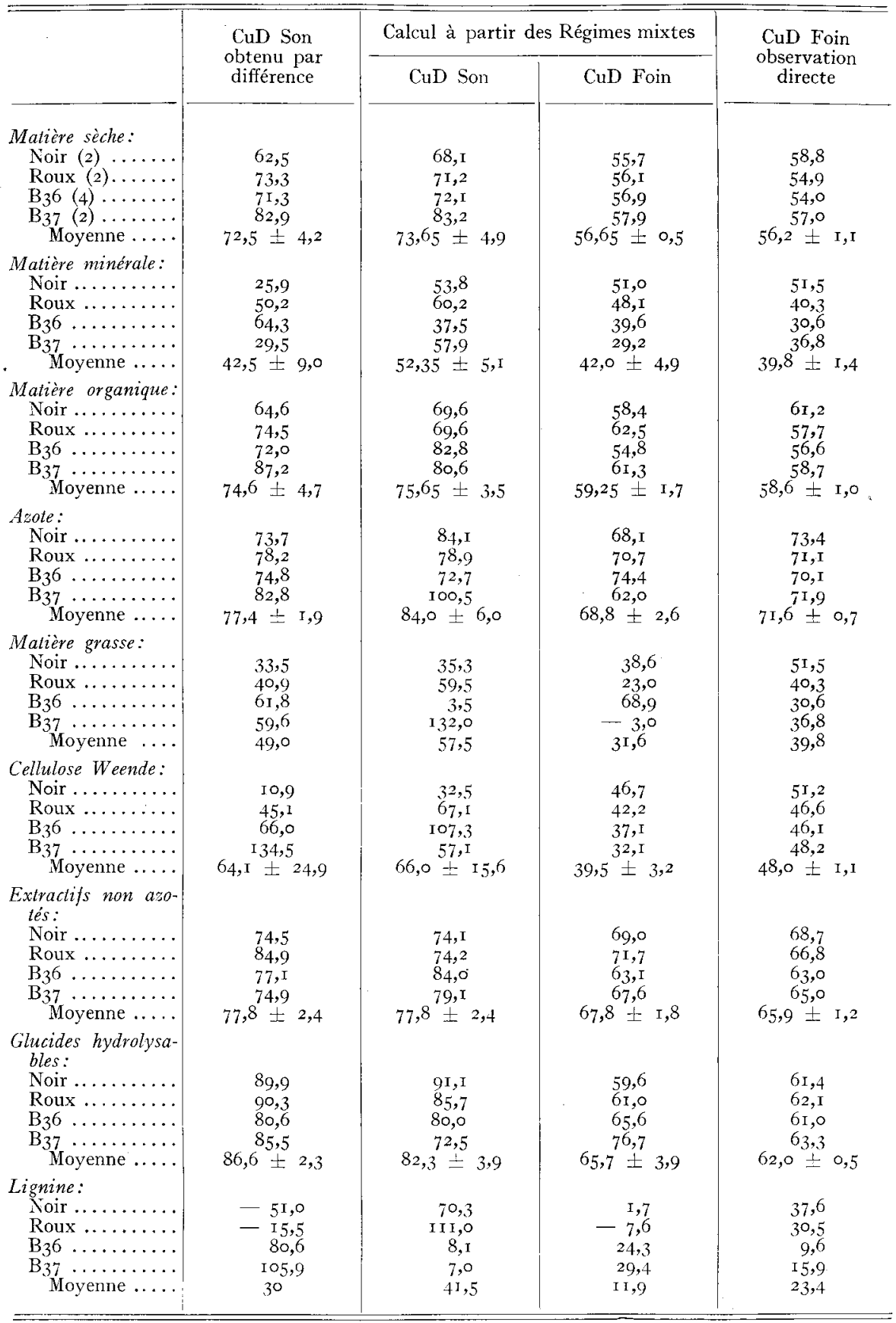


TABLEAU IV (swite)

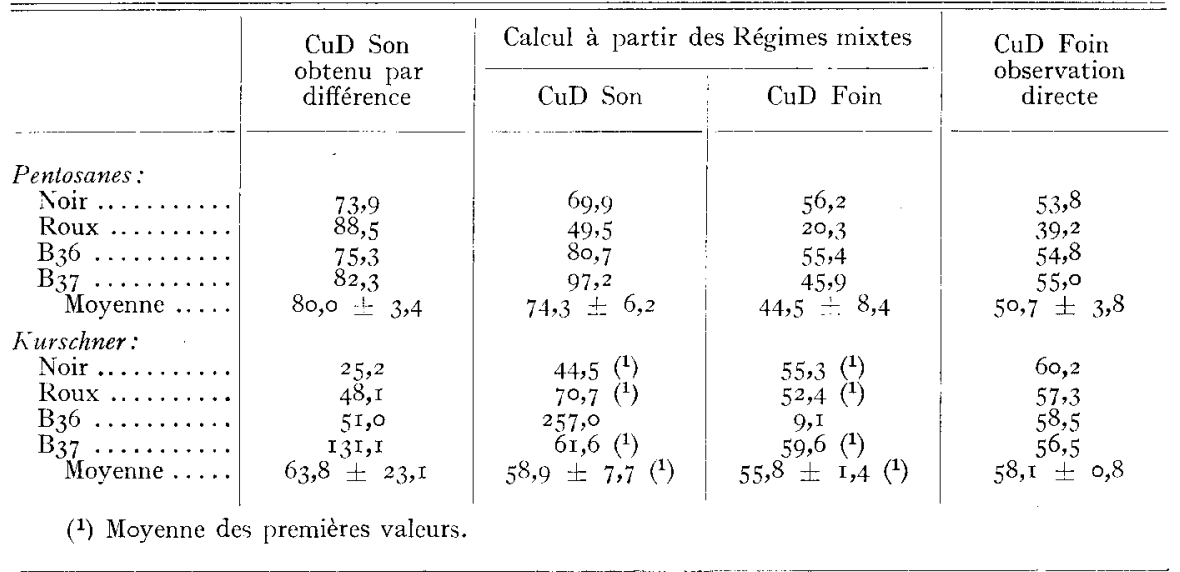

faibles que les moyennes de ces mêmes coefficients calculés par différence.

Cette amélioration de l'homogénéité se marque très nettement sur les éléments dont les résultats sont normalement très dispersés : cellulose WEENDE, cellulose KurSchNer, lignine.

Mais la comparaison entre les coefficients de digestibilité du foin, obtenus par calcul ou observés directement, met en évidence des variations importantes; en particulier le coefficient de digestibilité de la cellulose WEENDE est significativement plus faible lorsqu'il est calculé à partir des régimes mixtes que lorsqu'il est observé directement. Ce phénomène est normal, puisque 1'on sait que l'apport de sucres et d'amidon dans le régime d'un ruminant diminue la digestibilité des éléments cellulosiques. La digestibilité de la cellulose KuRschner et celle de la lignine sont affectées d'une façon analogue.

L'établissement de la droite caractérisant les variations du coefficient de digestibilité en fonction du pourcentage d'éléments semble intéressant à divers points de vue :

- les résultats obtenus sont un peu plus homogènes que ceux donnés par le calcul par différence.

- les résultats sont indépendants des valeurs trouvées par le régime de référence,

- il semble possible de mieux interpréter les phénomènes.

Remarquons cependant que, si nous ne possédons que 2 points caractéristiques crorespondant à 2 taux relativement proches, les erretrrs expérimentales sur chaque expérience, parfois assez importantes, pour les éléments chimiques présents en faible quantité (lignine) ou présentant des difficultés analytiques (matières grasses) peuvent entraîner 
l'inversion du signe mathématique de la pente de la droite, ce qui entraine de ce fait des erreurs grossières.

Pour apporter de la précision dans ce dernier cas, il faudrait presque obligatoirement étudier 3 pourcentages différents, pourcentages qui devraient être suffisamment différents les uns des autres, afin d'éviter les erreurs signalées précédemment.

Il ne nous paraît pas possible de conclure au remplacement d'un type de calcul par un autre, mais il nous semble surtout intéressant d'effectuer pour un même essai les 2 modes de calcul afin d'arriver à une interprétation aussi complète que possible des phénomènes étudiés.

Les coefficients de digestibilité que nous avons trouvés dans le son de froment concordent parfaitement avec ceux trouvés par d'autres auteurs (WATson et Coll. (9) et avec ceux cités dans la littérature (MoRRISON (IO).

\section{Résumé :}

Le calcul des coefficients d'utilisation digestive d'un aliment dans une ration mixte peut se faire, soit par la méthode classique dite de référence, soit en extrapolant pour la valeur roo la droite qui caractérise les variations des coefficients d'utilisation digestive des divers principes élémentaires du régime mixte en fonction du taux de ces derniers apportés par l'aliment étudié.

Les 2 méthodes appliquées à l'étude des coefficients de digestibilité du son chez les bovins et ovins conduisent à des résultats qui ne sont pas significativement différents.

\section{RÉFÉRENCES BIBLIOGRAPHIQUES}

(I) I EROY (A. M.), LERY (G.), ZELTER (Z.). - Contribution à l'étude de l'utilisation digestive des pulpes de betteraves desséchées par les porcs et les ruminants. Ann. Zoot., I952, 1, 29-49.

(2) Scrurch (A.). - Bestimmung, Berechnung und Signifikanz von verdanungskoeffizienten. Sonderdruck aus Heft I4/I954 der Schriftenreihe zur Fütterungslehre vom Fachverband der Futtermittelindustrie. Hamburg I.

(3) Gasnier (A.), VAchel (J. P.). - Recherches sur la méthode de mesure de la digestibilité d'un aliment. Condition d'emploi de cette méthode. Ann. Zoot., 1952, 1, I57 - I74.

(4) Gasnier (A.). - Prélèvements et analyses des échantillons d'aliments et produits destinés à l'alimentation des animaux. Communication de 1'Institut Professionnel de Contrôle et de Recherches Scientifiques des Industries de 1'Alimentation animale, I, Rue Santos-Dumont à Paris, I95I.

(5) Kurschner (K.), Hoffer (A.). - A new process for the determination of cellulose in wood and pulp. Fech. chem. Paper Zellstoff Fab., 26, p. I25-I39, I929, 31, p. I4, I934. 
(6) Mahood (J. A.), Cable (O. F.). - Chemistry of wood IV. - Analyses of Eucalyptus Globules and Pinus Monticola. J. Ind. Eng. Chem., 14, p. 933, I922.

(7) UNGER (E.), JAGER (R.). - Ueber Pentosanbestimmungen. Ber., 36, p. 1222,1903 .

(8) Bertrand (G.). - Composition et méthode d'analyse des tissus végétaux lignifiés. Ann. Fermentations, 1, p. 577-595, 1935.

(9) Watson (C. J.), Campbeli (J. A.), Davidson (W. M.), Robinson (C. H.), MUIR (G. W.). - Digestibility studies with ruminants. IX Associative digestibility of grains: wheat bran, gluten feed and soybean oil meal. Scientific Agriculture, 22, 1942.

(I0) Morrison. - Feed and Feeding $22^{\mathrm{e}} \mathrm{cd}$. Ithaca New York 1952. 